\title{
TRANSPOSITION OF THE SOLEUS INTO THE BED OF THE EXTENSOR DIGITORUM LONGUS MUSCLE IN THE RAT
}

\section{ERNEST GUTMANN* and BRUCE M. CARLSON**}

Institute of Physiology, CSA V, Prague 4, KRC (Czechoslovakia) and Department of Anatomy, University of Michigan, Ann Arbor, Mich. 48109 (U.S.A.)

(Received March 29th, 1978)

(Revised version received April 25th, 1978)

(Accepted April 26th, 1978)

\section{SUMMARY}

In 38 rats the slow soleus muscle was transposed, with its neurovascular bundle intact, into the bed of the fast extensor digitorum longus muscle. Transposed muscles underwent degeneration and regeneration like free muscle grafts. Both contractile and histochemical properties remained those of the soleus muscle, supporting the conclusion that the nerve to a slow muscle is pre-eminent in determining the functional and histochemical characteristics of the muscle.

Among the factors and tissue interactions that determine the structural, functional and metabolic properties of a muscle, the effects of the functional environment have proven to be among the most difficult to assess. The experimental method of muscle transpiosition [6] has facilitated analysis of these effects because it permits one to dissociate effects produced by the motor nerve to a mu scle from those that are erivironmentally induced.

Transposition of a muscle is accomplished by cutting both of its tendons and lifting the entire muscle, with it: motor nerve and associated vascular bundle, from its own bed and placing it into the bed of another muscle, which had been previously removed. This procedure differs from cross innervation [1], in which the muscle remains intact in its own bed and the motor nerve supply is exchanged with that of another muscle. In cross-transplantation, a muscle is : disconnected from its tendons of origin and insertion as well as its neurovascular supply and then freely grafted into the bed of another muscle [7].

\footnotetext{
*Deceased

**Address correspondence to: Bruce M. Carlson, Department of Anatomy, 462.2 Medical Sciences II, School of Medicine, University of Michigan, Ann Arbor, Mich. 48109 (U.S.A.)
} 
After this procedure, the muscle graft becomes reinnervated by the nerve to the muscle in whose bed it was placed, and it also becomes anacomically integrated into the functional environment of the host muscle bed.

This experiment was carried out on 38 two-month old male rats of the inbred AVN strain that is maintained at the Institute of Physiology in Prague. The fast extensor digitorum longus (EDL) muscle was removed from one leg, and the stump of its motor rerve was embedded into another muscle. Then the proximal and distal tendons of the slow soleus muscle were cut and the muscle was carefully moved over into the bed of the EDL so that its neurovascular bundle remained intact. The proximal and distal tendons of the transposed soleus were sutured to the corresponding tenclon stumps of the EDL, and the overlying fascia and the skin were sutured closed in layers. This experiment consisted of two series: one in which the soleus muscle was transposed as described above and another in which the soleus was injected with $0.75 \%$ Marcaine (Winthrop) before transposition. Injection with Marcaine produces a nearly complete degeneration anc subsequent regeneration of the muscle fibers within the muscle $[2,8]$.

At intervals of $7,14,30$ and 60 days after transposition, both the transposed and the contralateral normal soleus muscles were removed from anesthetized rats. Th 2 muscles were placed into an oxygenated culture medium and set up for direst electrical stimulation and recording of contractile properties in vitro [3]. After determination of the optimal resting tension, the following contractile characteristics were recorded: twitch and tetanic tensions, latency period, full contraction time (time to peak tension) and half relaxation time. After analysis of contractile properties, the muscles were examined histochemically for succ inic dehydrogenase (SDH) and myofibrillar adenosine triphosphatase (ATPase) activity.

There was relatively little difference between the series in which the normal soleus ruscle was transposed and that in which the muscle was first treated with Marcaine. Therefore only data from the former series will be presented here. Ii both groups there was morphological evidence of substantial degeneration and subsequent regeneration of muscle fibers within the transposed muscles It is apparent that whatever vascular supply was preserved in the neurovascular bundle was not sufficient to preserve the structural integrity of the muscle fioers in the transposed normal soleus muscles.

Contractile properties (Table I). The low twitch (Tw. T.) and tetanic tensions (Tet. T.) of the transposed soleus muscles reflected the breakdown and subsequent regeneration of muscle fibers in the transposed muscles. In these experiments the muscles remained weak, a characteristic of regenerating and fi:eply grafted soleus muscles in the rat [5]. The iull contraction time (FCT) and half relaxation time (HRT) remained slow and did not differ significantly from the values of the contralateral soleus (Table I). The full contraction time of the normal E.DL and also 30- or 60-day free grafts of this muscle is three times faster than that of the transposed soleus [5].

Histochemical properties. Sections through 7- and 14-day transposed muscles 


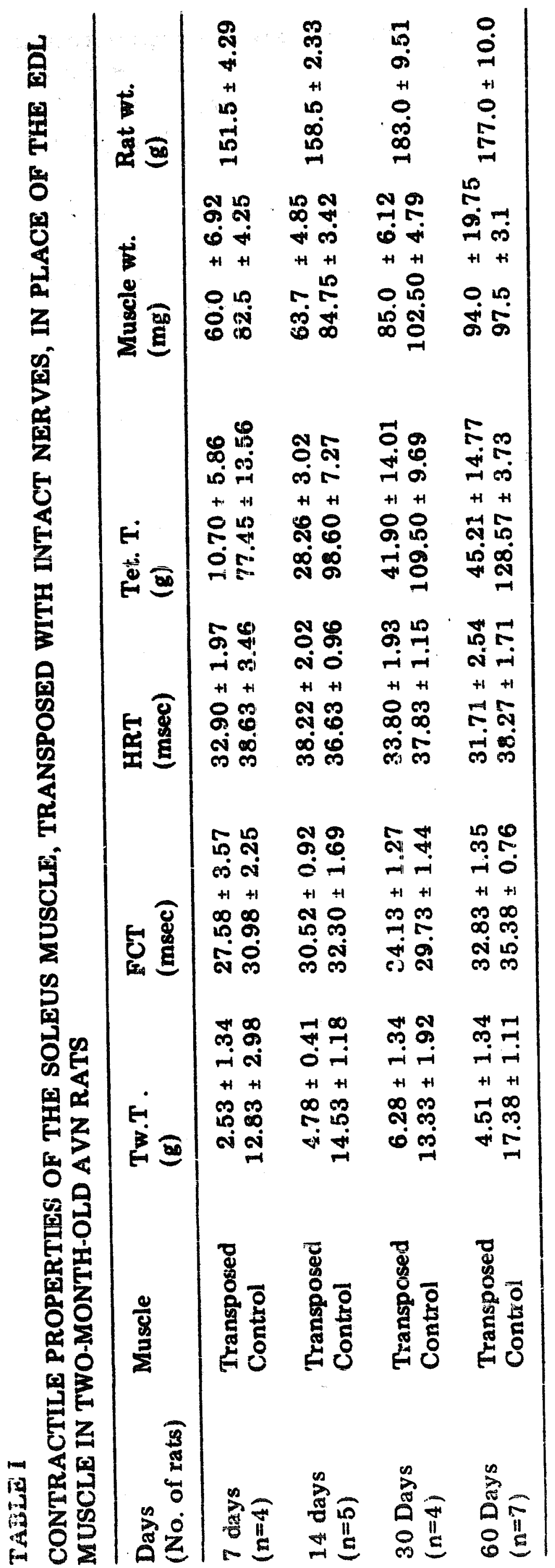


showed clear evidence of degeneration and regeneration in the central areas of transposed muscles. Surviving peripheral muscle fibers retained their histochemical staining characteristics and remained much larger than the newly regenerating muscle fibers, which stained uniformly $f(r$ both SDH and ATPase activity. By 30 days dark and light muscle fibers were clearly distinguishable in ATPase preparations, and sections stained for SDH activity showed heterogeneous staining, as well. Sixty day muscles appeared to be stable with respect to histochemical staining characteristics. The outstanding characteristic of these muscles was their similarity to the contralateral control soleus muscles (Fig. 1). The transposed muscles retained the 'checkerboard' or mosaic pattern of staining. In only restricted areas were there small 'type-groups' of homogeneously staining fibers. SDH activity in the transposed muscles was high and the overall staining pattern resembled closely that of the normal soleus muscle.
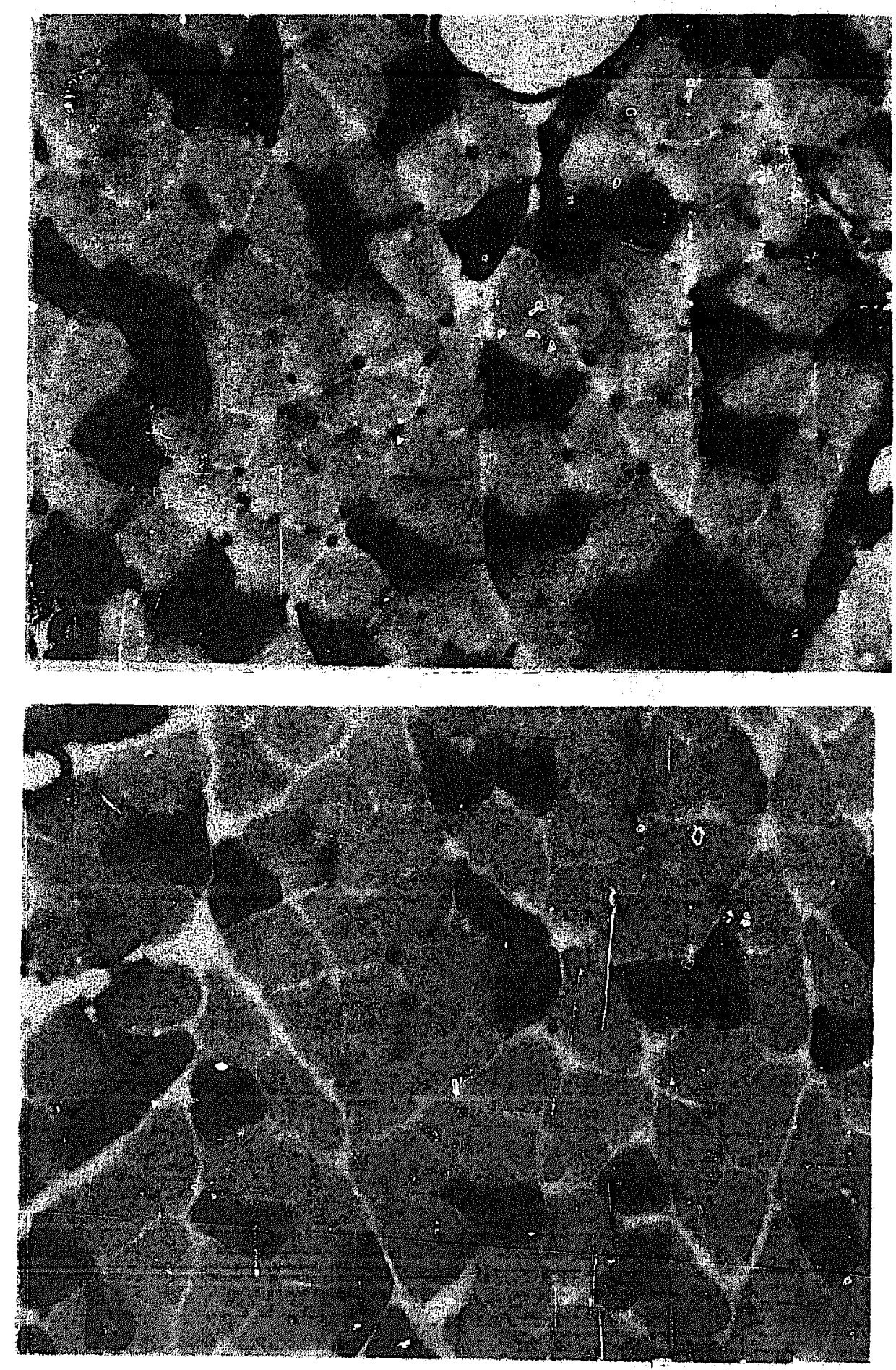

Fig. 1. Myofibrillar ATPase preparations of a normal soleus muscle (A) and a soleus transposed in $: 0$ the bed of the EDL for 60 days (B). The staining pattern characteristic of the normal soleus is retained in the transposed muscle. $\times 250$. 
It is well known from studies of both cross union of nerves [1] and crosstransplantation of muscles [7] that the quality of the motor nerve determines to a large extent the contractile and histochemical properties of a muscle. In these experimental models the conversion between rast and slow properties is not always complete, and the degree of influence of the local functional environment has not been entirely clear.

Earlier, Gutmann [6] transposed the EDL muscle into the bed of the soleus in rats. He found that the contractile properties of the transposed muscles remained fast, but that within the transposed EDL muscles the histochemical pattern of SDH activity became converted to a predominantly high oxidative type,.like that of the soleus muscle. In the present study not only did the the contractile properties and staining pattern of ATPase activity retain the characteristics of the slow soleus muscle, but the histochemical pattern of oxidative activity, as indicated by SDH staining, remained that of the soleus muscle. After cross-transplantation of the soleus muscle there was an almost complete conversion of both the contractile and histochemical properties to those of the EDL, into the bed of which the soleus was grafted [7]. Thus, the properties of the transposed soleus muscles remained surprisingly independent of the bed of the fast muscles into which they were placed, suggesting that in this case the quality of the motor nerve is the dominant force in determining the functional characteristics of the muscle.

As was the case of the EDL muscle grafted into the bec of the soleus [6], this transposition model should be looked upon as a free graft with an attached nerve, because after transposition considerable degeneration and subsequent regeneration occur in a pattern closely resembling that of a true free graft $[4,5]$. The outstanding difference is the preservation of the original histochemical mosaic of muscle fiber types, reflecting the dispersal of the muscle fibers within the motor unit. In a typical free muscle graft, in which the nerve is also cut, the muscle fibers of a mature graft are clustered into similar type groups, a phenomenon interpreted by Karpati and Engel [9] as indicative of innervation. The other major difference between the transposed and the freely grafted soleus muscle was the retention of distinct differences in staining characteristics (especially ATPase) by the peripheral surviving nuscle fibers and the differentiation of clearly distinct histochemical fiber types within the regenerating muscle fibers as early as $\mathbf{3 0}$ days. In a free graft the histochemical differences among the denervated surviving muscle fibers become diminished, and the differentiation of muscle fiber types in areas of regeneration occurs about a week later.

It is apparent that the motor nerve to the soleus is able to maintain the functional and histochemical differentiation of the muscle despite the muscle's being transposed into a foreign functional environment.

\section{ACKNOWLEDGEMENTS}

Supported by grants from the Muscular Dystrophy Association and $\mathrm{NIH}$ 
(NS 13116), as well as a scientific exchange between the Academies of Sciences of Czechoslovakia and the United States. Thanks are due to Mrs. A. Herbrychovd for expert technical assistance.

\section{REFIERENCES}

1 Juller, A.J., Eccles, J.C. and Eccles, R.M., Interaction between motoneurones and ruscles in respect of the characteristic speeds of their responses, I. Physiol. (Lond.), $150(1960) 417-439$.

2 Carlson, B.M', A quantitative study of muscle fiber survival and regeneration in normal, predenervated ars Marsaine-treated free muscle grafts in the rat, Exp. Neurol, 52 (1976) 421-432.

3 Carlson, B.M. and Gutmann, E., Development of contractile properties of minced muscle regenerates in the rat, Exp. Neurol., 36 (1972) 239-249.

4 Carlson, B.M. and Gutmann, E., Regeneration in frete grafts of normal and denervated muscles in the rat: Morphology and histochemistry, Anat. Rec., 183 (1975) 47-62.

5 Carlson, B.M. and Gutmann, E., Regeneration in grafts of normal and denervated rat Inuscles. Contractile properties, Pflugers Arch., 353 (1975) 215-225.

6 Gutmann, E., Effect of transposition on contractile and histochemical properties of muscle, Neuroscience Letters, 2 (1976) 273-277.

7 Gutmann, E., and Carlson, B.M., Contractile anu histochemical properties of regenerating cross-transplanted fast and slow muscles in the rat, Pflugers Arch., 353 (1975) 227-239.

8 Hall-Craggs, E.C.B., Rapid degeneration and regeneration of a whole skeletal muscle following treatment with bupivacaine (Marcaine), Exp. Neurol., 43 (1974) 349-358.

9 Karpati, G., and Engle, W.K., "Type Grouping" in skeletal muscles after experimental reinnervation, Neurclogy (Minneap.), 18 (1968) 447-455. 